\title{
GESTIÓN POR PROCESOS: REGULARIDADES METODOLÓGICAS Y COMPORTAMIENTO DE SU IMPLEMENTACIÓN
}

\section{MANAGEMENT \\ BY \\ PROCESSES: \\ METHODOLOGICAL \\ REGULARITIES \\ AND BEHAVIOR OF ITS IMPLEMENTATION}

\author{
(iD) Carlos Eugenio Vega Moreno ${ }^{1}$ (iD Lizbeth Dora Briones Pereyra $^{1}$ (iD Mendoza Corpus Carlos Alfredo \\ 1Universidad Nacional del Santa, Ancash, Perú \\ Correspondencia: \\ Dr. Carlos Eugenio Vega Moreno \\ cvega@uns.edu.pe
}

\section{RESUMEN}

La gestión por procesos constituye una importante herramienta para el logro de resultados superiores en cuanto a la eficacia y eficiencia en las organizaciones. Pese a las múltiples y variadas propuestas metodológicas para su implementación, la necesidad de contar con referentes científicamente validados para investigaciones sobre el tema condujo a esta investigación, en la que se definió como objetivo realizar un estudio del estado del arte de la gestión por proceso en cuanto a regularidades metodológicas y comportamiento de su implementación. El desarrollo de la investigación se sustentó en una revisión documental aplicando como métodos, el análisis-síntesis y la inducción-deducción. Se reconocieron como principales resultados, los tipos de organizaciones más intervenidas en la implementación de esta tecnología de gestión y la identificación de los momentos metodológicos que con mayor frecuencia se emplearon en las propuestas consultadas. Se concluyó que, existen suficientes fuentes bibliográficas de rigor científico que demuestran la importancia que advierte la gestión por procesos para lograr mejores resultados en cualquier tipo de organización; que la mayoría de las metodologías estudiadas responden a intervenciones realizadas a empresas, lo que presupone la importancia que para estas organizaciones empresariales representa el incremento de la productividad y la eficiencia que promueve esta tecnología; las regularidades identificadas en los momentos metodológicos constituyen referentes de valor para nuevas investigaciones, al estar científicamente demostrada su validez teórica y práctica.

Palabras clave: gestión por proceso, metodologías de gestión por proceso, implementación de la gestión por procesos

\section{ABSTRACT}

Management by processes is an important tool for achieving superior results in terms of effectiveness and efficiency in organizations. Despite the multiple and varied methodological proposals for its implementation, the need to have scientifically 
validated references for research on the subject led to this research, in which the objective was to carry out a study of the state of the art of process management in regarding methodological regularities and behavior of its implementation. The development of the research was based on a documentary review applying as methods, analysis-synthesis and induction-deduction. The main results were recognized, the findings regarding the types of organizations most involved in the implementation of this management technology and the identification of the methodological moments that were most frequently used in the proposals consulted. It was concluded that, there are sufficient bibliographic sources of scientific rigor that demonstrate the importance of process management to achieve better results in any type of organization; that most of the methodologies studied respond to interventions made to companies, which presupposes the importance that for these business organizations represents the increase in productivity and efficiency promoted by this technology; the regularities identified in the methodological moments constitute valuable referents for new investigations, since their theoretical and practical validity has been scientifically demonstrated.

Key words: management by process, management methodologies by process, implementation of management by processes

\section{INTRODUCCIÓN}

Ante un mercado complejo y cambiante, las organizaciones requieren obtener buenos resultados que le garanticen su competitividad y supervivencia. Para ello necesitan gestionar sus actividades y recursos orientando la consecución de estos buenos resultados a un cliente cada vez más exigente. En este contexto deberán adoptar también herramientas y metodologías novedosas científicamente demostradas que le permitan reconfigurar su sistema de gestión y alcanzar tales propósitos entre las que aparece la gestión por procesos. Gestión por procesos, dirección por proceso o gestión con enfoque de proceso, constituyen una familia de conceptos que expresan igual esencia en sus definiciones y el alto significado que se le confiere a garantizar el funcionamiento efectivo de los procesos.

Surgió de la necesidad de contar con un sistema de control que posibilitara la toma de decisiones basado no solo en el análisis económico - financiero, sino que lograra una valoración integral de la gestión y sirviera de herramienta en la identificación de las desviaciones en los procesos; así como, de la necesidad de hacer empresas eficientes que respondieran a las exigencias crecientes de los clientes (Pérez et al., 2017).
Su propósito es alinear los procesos de negocio de la organización a los objetivos y necesidades de los clientes estratégicos (Ponce, 2016). Constituye nueva filosofía de gestión donde prevalece una orientación hacia el cliente; se sientan las bases para garantizar la optimización y racionalización en el uso de los recursos con criterios de eficiencia y eficacia; además que, posibilita un mayor visualización de las relaciones internas de la organización; se reducen costos y se logran identificar con mayor acierto aquellos asociados a la mala calidad, ayudando todo ello a la toma de decisiones eficaces, tal y como han defendido autores como: (Pernett, 2004), (Saballo, 2005), (Ponjuán et al., 2005), (Pérez, 2010), (Carrasco, 2011), (Llanes et al., 2014).

Se apunta como una de las herramientas de mejora de la gestión más efectivas para todos los tipos de organizaciones constituyendo, además, uno de los momentos iniciales e indispensables del proceso de implementación del sistema de gestión de la calidad (Calderon, 2018). Busca reducir la variabilidad que aparece habitualmente cuando se producen o prestan determinados servicios y trata de eliminar las ineficiencias asociadas a la repetitividad de las acciones o actividades y al 
consumo inapropiado de recursos (Hernández et al., 2013).

Su implementación supone reordenar los flujos de trabajo de forma de reaccionar con más flexibilidad y rapidez a los cambios y en la búsqueda del ¿por qué? y ¿para quién? se hace el trabajo (Medina et al., 2019). Además, abarca áreas clave como la garantía de calidad, los sistemas, estructura, estrategia, proceso impulsado por los clientes, gestión del rendimiento y la resolución de problemas con el propósito de mejora continua. (Ponce, 2016).

Asumirla significa determinar y gestionar numerosas actividades relacionadas entre sí, a fin de aumentar la satisfacción del cliente mediante el cumplimiento de los requisitos establecidos, de igual forma, mejora la comunicación interna, la eficiencia y eficacia de los procesos, brinda un incremento en la satisfacción de los empleados y mejora la posición competitiva de la empresa, cualidades éstas expresadas por autores como (Zaratiegui, 1999); (Ponjuán et al., 2005), (Alfonso et al., 2011), (Cejas, 2012), (Gimer, 2012), (Hernández et al., 2013); (Pérez et al., 2017); (Hernández et al., 2018), (Medina et al., 2019).

Para lograr el establecimiento de un enfoque a procesos se parte de la definición de los procesos clave, de apoyo y estratégicos, siendo estos últimos los que organizan planifican, dirigen y controlan los demás procesos (Quintana et al., 2013). Se requiere además de un cómo actuar que facilite su implementación de manera loable, de allí que autores como (Carrasco, 2011), ( Hernández et al., 2013), (Acosta, 2015), (Ponce, 2016), (Morell, 2017), (Rodríguez, 2017), (Betancourt, 2018), (Calderon, 2018), entre otros, realizaran importantes aportes metodológicos en este sentido.

Las múltiples propuestas metodológicas encontradas para implementar la gestión por procesos, y su diversidad en cuanto a tipos de organizaciones objeto de intervención y los momentos metodológicos para su aplicación ante cada contexto suscitaron esta investigación, la que se propuso como objetivo realizar un estudio del estado del arte de la gestión por procesos en cuanto a regularidades metodológicas y comportamiento de su implementación.

\section{MATERIALES Y MÉTODOS}

La investigación se llevó a cabo con una exhaustiva revisión documental utilizando como principales métodos el análisis-síntesis y la inducción-deducción.

El estudio se realizó con dos grandes propósitos, de modo que, el primero, se dirigió a conocer los tipos de organizaciones objetos de estudio intervenidos con la implementación de esta tecnología de gestión y qué comportamiento manifestaban en este sentido; el segundo, para determinar las regularidades de momentos metodológicos considerados en estas propuestas y que pudieran servir de importantes referentes para nuevas investigaciones.

Para realizar el estudio de manera general se utilizaron un total de 37 fuentes bibliográficas con validez científica que incluyeron libros, tesis de maestrías y doctorados y artículos científicos publicados en revistas indexadas. Su análisis posibilitó distinguir las esencias y elementos que caracterizan la gestión por procesos y en cuáles de estas fuentes se realizaron aportes metodológicos, lo que contribuyó a al primer propósito de la investigación.

A partir del análisis de estas fuentes se elaboraron dos matrices auxiliares: la Tabla 1 , a fin de identificar qué tipo de organizaciones eran objetos de intervención para implementar la gestión por procesos; y la Tabla 2 para determinar la regularidad de los pasos para implementar esta tecnología de gestión.

Para elaborar la Tabla 1 , se realizó un levantamiento de todas las fuentes autorales consultadas que exponían aportes metodológicos y los títulos de sus propuestas. Estas se listaron en la primer y segunda columna, mientras se desplegaron 
otras columnas para señalar los tipos de organizaciones intervenidas, según se fueron identificando en el estudio. Los datos registrados en esta Tabla 1 , devinieron en la identificación de los países orígenes de estas metodologías o de su implementación, en la identificación de los tipos de organizaciones en que éstas habían sido aplicadas, y en el comportamiento de esta implementación de acuerdo al tipo de organización.

Con el fin de, alcanzar el segundo propósito de esta investigación de identificar la regularidad de los momentos metodológicos para implementar la gestión por procesos, fue necesario realizar un análisis pormenorizado de las esencias de las fases, etapas y/o pasos declarados por las fuentes registradas en la Tabla 1: (Comité Técnico ISO/TC 176, 2003), (Arrascaeta, 2005), (Saballo, 2005), (Beltrán et al., 2009), (Mallar, 2010), (Carrasco, 2011), (Bravo, 2012), (Rivero, 2013), ( Hernández et al. 2013), (Alonso et al., 2013), (Acosta, 2015), (Ponce, 2016), (Pérez et al., 2017), (Rodríguez, 2017), (Morell, 2017), (Betancourt, 2018), (Calderon, 2018), (Hernández et al., 2018), (Rivero, 2018), (Medina et al., 2019) y (Eneque et al., 2020).

Para llevar a cabo este análisis, se elaboró una Tabla auxiliar en la que se destinó una fila para cada metodología desplegando por columnas los momentos metodológicos por ésta declarados. Con estos datos se realizó un proceso de análisis exhaustivo de cada fase, etapa y/o paso que permitió descubrir sus esencias para luego establecer comparaciones con los momentos metodológicos de las siguientes propuestas y poder sintetizarlos. Como resultado de este proceso analíticocomparativo se excluyeron los pasos que fueron considerados solamente por una fuente y se realizaron cinco grandes agrupaciones de pasos, fases, etapas y/o pasos afines por su esencia convirtiéndose en los momentos metodológicos a analizar.

La Tabla 2, se elaboró listando por filas las cinco agrupaciones, devenidas en momentos metodológicos, mientras que por columnas se registraron las 21 fuentes autorales con aportes metodológicos. Con esta estructura de matriz se fueron identificando, para cada fuente, la correspondencia, según su esencia, entre las fases, etapas y/o pasos expuestos en su metodología y los momentos metodológicos a los que se habían arribado. Los datos registrados en la Tabla, posibilitaron identificar la regularidad de los momentos metodológicos en las 21 propuestas investigadas.

\section{RESULTADOS Y DISCUSIÓN}

Como primer resultado de la investigación se reconoce la identificación de 21 fuentes bibliográficas con aportes metodológicos y su implementación en los diferentes contextos organizacionales, tal y como muestra la Tabla 1.

Como segundo resultado, se reconoce el descubrimiento de los países en que estas metodologías fueron aplicadas o concebidas. Se encontraron propuestas provenientes de países como Perú, Chile, Colombia, España, Cuba, México y Venezuela, lo que evidencia el reconocimiento que a nivel global se le concede a esta tecnología de gestión.

Se encontró también que, la mayoría de estas metodologías se acompañaron con resultados de su contextualización en las organizaciones intervenidas, lo que posibilitó identificar los tipos de organizaciones que fueron objeto de estudios y qué comportamiento mostraba la implementación de esta tecnología de gestión, lo que fue también registrado en la Tabla 1. 
Tabla 1

Especies de plantas, peso del material seco y volumen de alcohol utilizados para la obtención de los extractos en el ensayo

Autores

Tipos de organizaciones objetos de intervención
(a)
(b)
(c)
(d)
(e)
(f) $\quad(g)$

(Carrasco, 2011)

(Hernández et al., 2013)

(Acosta, 2015)

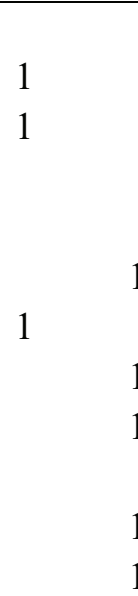

(Rodríguez, 2017)

1

(M. E. Rivero, 2018)

(Morell, 2017)

(Hernández et al., 2018)

(Calderon, 2018)

(Betancourt, 2018)

(Medina et al., 2019)

(Eneque et al., 2020)

(Ponce, 2016)

(Alonso et al., 2013)

(Beltrán et al., 2009)

(Arrascaeta, 2005)

(Bravo, 2009)

(Mallar, 2010)

(Comité Técnico ISO/TC 176, 2003)

(Saballo, 2005)

(Rivero, 2013)

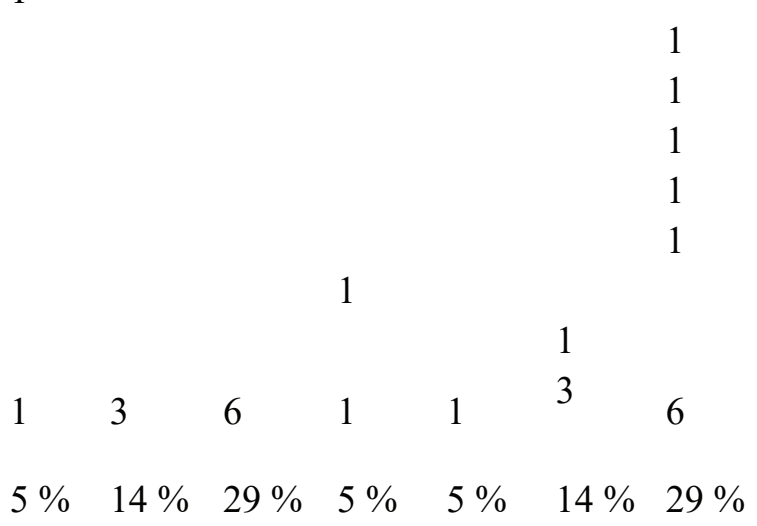

Leyenda: a) Educación, b) Salud, c) Empresas, d) Hotelería e) Administración Pública, f) Otros tipos de organizaciones, g) Aplicable a cualquier tipo de organización

Con el análisis de estos datos se arribó a que fueron intervenidas organizaciones de tipo: empresa, instituciones de salud, de educación, organizaciones de la administración pública y establecimientos hoteleros, aunque también se encontraron estudios en dos organizaciones de tipo funcional, mientras que seis de las metodologías resultaban aplicables a cualquier tipo de organización por su grado de generalidad. La Figura 1, representa este comportamiento. 


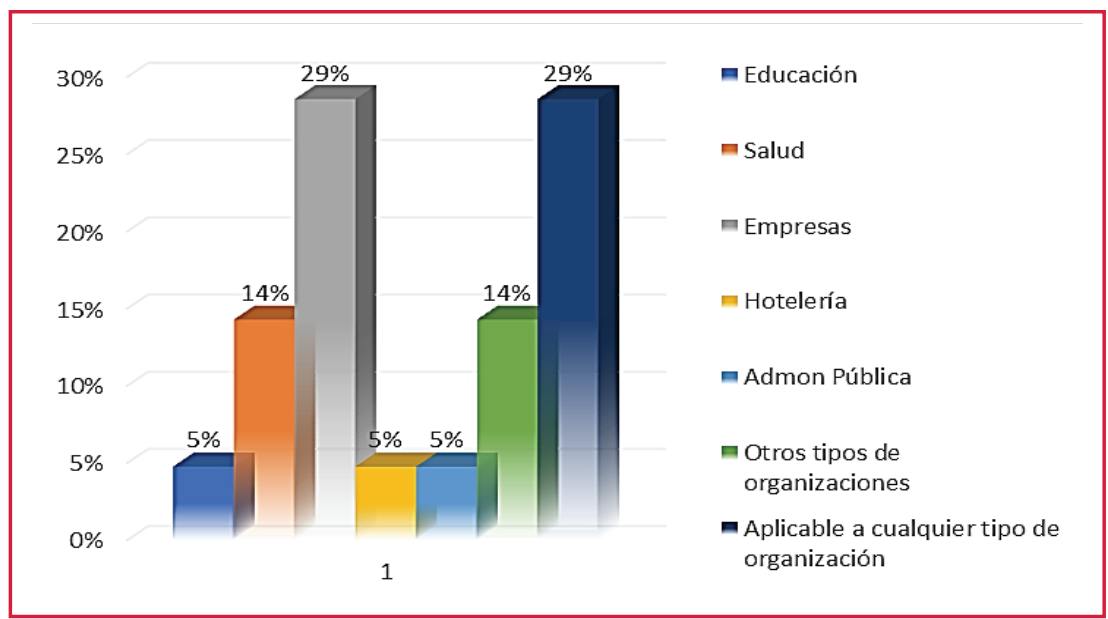

Figura 1. Comportamiento de la implementación de la gestión por procesos por tipo de organizaciones

Según muestra la Figura 1 y el análisis de la Tabla 1, prevaleció la empresa como objeto de estudio con seis (29\%) de las 21 propuestas consultadas: Ponce (2016), Morell (2017), Betancourt (2018) y Pérez et al. (2017), en empresas productivas, mientras que Calderón (2018) y Eneke \& Tello (2020), lo aplicaron en empresas de servicios.

Se descubrió además que, algunos establecimientos de salud también fueron objeto de intervención. En este sector se encontraron tres estudios: Hernández et al. (2013) y Acosta (2015), en instituciones hospitalarias y Hernández et al. (2018), en un hogar de ancianos, los que adecuaron su metodología de implementación en hospitales para un hogar de ancianos. Ello representó un $14 \%$ de las intervenciones realizadas.
Mientras, idénticas cifras mostraron las agrupación realizada en Otros tipos de organizaciones como el caso de la Fundación Desayunitos Creando Huella descrita por (Rodríguez, 2017) y el de la revista científica Retos de la Dirección reportada por (Rivero, 2018).

Por últimos se observó que seis (29\%) de las 21 fuentes, entre los que se encontraban (Comité Técnico ISO/TC 176, 2003), (Arrascaeta, 2005), (Narbarte et al., 2007), (Amozarrain, 2009), (Bravo, 2009) y (Mallar, 2010), ofrecieron metodologías generales aplicables a cualquier tipo de organización y no declararon objetos de estudio.

En cuanto al estudio de la regularidad de los momentos metodológicos, el proceso analítico-comparativo dio lugar a la Tabla 2. 


\section{Tabla 2}

Análisis de la regularidad de los momentos metodológicos según fuentes autorales

\begin{tabular}{|c|c|c|c|c|c|c|c|c|c|c|c|c|c|c|c|c|c|c|c|c|c|c|}
\hline \multirow{2}{*}{$\begin{array}{l}\text { Momentos } \\
\text { metodológicos }\end{array}$} & \multicolumn{22}{|c|}{ Autores según leyenda } \\
\hline & 1 & 2 & 3 & 4 & 5 & 6 & 7 & 8 & 9 & 10 & 11 & 12 & 13 & 14 & 15 & 16 & 17 & 18 & 19 & 20 & 21 & $\%$ \\
\hline $\begin{array}{lr}\text { Iniciación } & \text { del } \\
\text { proceso } & \text { de }\end{array}$ & & & & & & & & & & & & & & & & & & & & & & \\
\hline implementación & $\mathrm{x}$ & $\mathrm{x}$ & $\mathrm{x}$ & $\mathrm{x}$ & $\mathrm{x}$ & & $\mathrm{x}$ & $\mathrm{x}$ & $\mathrm{x}$ & $\mathrm{x}$ & & $\mathrm{x}$ & $\mathrm{x}$ & $\mathrm{x}$ & & & & $\mathrm{x}$ & & $\mathrm{x}$ & $\mathrm{x}$ & 71 \\
\hline $\begin{array}{l}\text { Definición } \\
\text { descripción }\end{array}$ & & & & & & & & & & & & & & & & & & & & & & \\
\hline los procesos & $\mathrm{x}$ & $\mathrm{x}$ & $\mathrm{x}$ & $\mathrm{x}$ & $\mathrm{x}$ & $\mathrm{x}$ & $\mathrm{x}$ & & & $\mathrm{x}$ & $\mathrm{x}$ & $\mathrm{x}$ & & $\mathrm{x}$ & $\mathrm{x}$ & $\mathrm{x}$ & & $\mathrm{x}$ & $\mathrm{x}$ & & $\mathrm{x}$ & 76 \\
\hline Análisis de & & & & & & & & & & & & & & & & & & & & & & \\
\hline $\begin{array}{l}\text { relevancia } \\
\text { clasificación }\end{array}$ & $\mathrm{x}$ & & & $\mathrm{x}$ & & & $\mathrm{x}$ & $\mathrm{x}$ & $\mathrm{x}$ & & $\mathrm{x}$ & $\mathrm{x}$ & & & & & $\mathrm{x}$ & $\mathrm{x}$ & $\mathrm{x}$ & $\mathrm{x}$ & & 52 \\
\hline $\begin{array}{l}\text { Descripción de } \\
\text { los procesos }\end{array}$ & & $\mathrm{x}$ & $\mathrm{x}$ & & & & & & $\mathrm{x}$ & $\mathrm{x}$ & & & & $\mathrm{x}$ & $\mathrm{x}$ & $\mathrm{x}$ & $\mathrm{x}$ & & & $\mathrm{x}$ & $\mathrm{x}$ & 48 \\
\hline $\begin{array}{l}\text { Seguimiento, } \\
\text { control y mejora }\end{array}$ & $\mathrm{x}$ & & & & $\mathrm{x}$ & $\mathrm{x}$ & & $\mathrm{x}$ & $\mathrm{x}$ & $\mathrm{x}$ & $\mathrm{X}$ & $\mathrm{x}$ & & $\mathrm{x}$ & $\mathrm{x}$ & $\mathrm{x}$ & $\mathrm{x}$ & $\mathrm{x}$ & $\mathrm{x}$ & $\mathrm{x}$ & $\mathrm{x}$ & 76 \\
\hline
\end{tabular}

\section{Leyenda:}

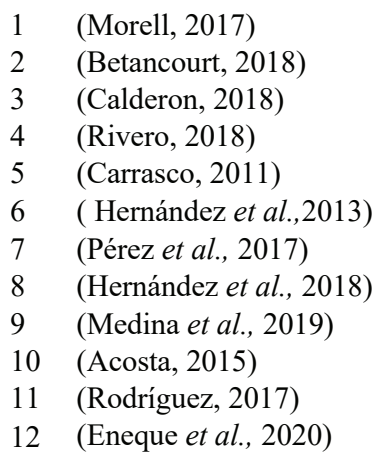

13 (Ponce, 2016)

14 (Alonso et al., 2013)

15 (Beltrán et al., 2009)

16 (Arrascaeta, 2005)

17 (Bravo, 2012)

18 (Mallar, 2010)

19 (Comité Técnico ISO/TC 176, 2003)

20 (Saballo, 2005)

21 (Rivero, 2013)

Asimismo, los datos de esta Tabla posibilitaron obtener los resultados que se muestran en la Figura 2.

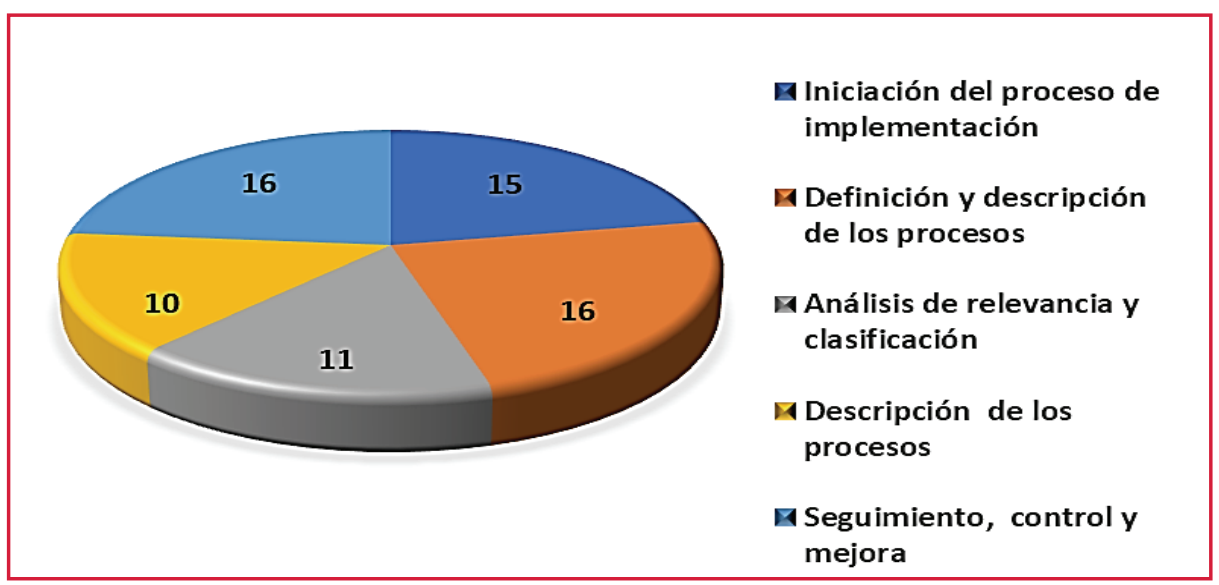

Figura 2. Comportamiento de los momentos metodológicos según propuestas consultadas 
De acuerdo a la Figura 2 y Tabla 2, tanto en la definición y descripción de los procesos, como seguimiento, control y mejora, fueron momentos metodológicos cada uno tomado en cuenta por el $76 \%$ de las propuestas metodológicas estudiadas.

Este resultado manifiesta, por una parte, la importancia que los autores le confieren a la definición acertada de los procesos y a su descripción. Constituye un momento esencial durante la implementación de la gestión por procesos pues se encarga de realizar el estudio de todos los procesos de la organización, sus características y sus relaciones con otros procesos, analiza el comportamiento de cada uno y de todos en su conjunto; así como, su papel en la organización, y finalmente se clasifican estos procesos de acuerdo a su misión en la organización para diseñar el mapa de procesos.

Por su parte el Seguimiento, control y mejoras, como otro momento de relevancia, refleja la necesidad que identificaron sus autores de establecer mecanismos sistemáticos de control sobre los procesos; así como, de las acciones de mejora para corregir las desviaciones o prevenir la ocurrencia de no conformidades. Definir qué indicadores medir y, cómo controlarlos, además de, cómo mejorar los procesos quedaron incluidos en este tránsito metodológico hacia la gestión por procesos.

La iniciación del proceso de implementación resultó el tercer momento más tomado en cuenta por las diferentes propuestas metodológicas con un $71 \%$ de citación, lo que denota la importancia conferida a la creación de las condiciones para enfrentar la implementación de esta tecnología de gestión. En ellas se incluyeron creación y preparación de grupos de trabajo, planificación de tareas a desarrollar y diagnósticos organizacionales entre los más referidos.

Igualmente, de importante resultó la Definición de los procesos más relevantes con una presencia cada uno en el $52 \%$ de las metodologías y la Descripción de los procesos, con una representatividad del 48 $\%$ de las propuestas. Con ello se garantiza la selección estratégica de los procesos de mayor impacto en la organización, el diseño - rediseño adecuado de cada proceso en estudio, su articulación con otros procesos, y el alineamiento de estos con los objetivos estratégicos de la organización y con los requerimientos y expectativas de los clientes.

\section{CONCLUSIONES}

- Se encontraron suficientes fuentes bibliográficas de rigor científico que demuestran la importancia que advierte la gestión por procesos para lograr mejores resultados en la gestión de cualquier tipo de organización al mostrar con claridad dónde se crea valor en la organización y cómo se produce la articulación entre esta y los clientes, además que proporciona un mayor alineamiento entre los objetivos estratégicos de la organización y las necesidades y expectativas de los clientes.

- La investigación posibilitó identificar que el mayor por ciento de las metodologías estudiadas aplicadas en organizaciones responde a intervenciones realizadas a empresas, lo que presupone la importancia que para estas organizaciones empresariales representa el incremento de la productividad y la eficiencia, aspectos que favorece de manera significativa gestionar por procesos.

- Las regularidades identificadas en los momentos metodológicos considerados en las metodologías para la implementación de la gestión por procesos que fueron consultadas en esta investigación, constituyen referentes de valor para nuevas investigaciones, al estar científicamente demostrada su validez teórica y práctica.

\section{REFERENCIAS BIBLIOGRÁFICAS}

Acosta, E. (2015). Guía metodológica para implementar el enfoque basado en proceso en instituciones hospitalarias cubanas [Tesis de posgrado]. Universidad de Camagüey Ignasio Agramonte Loynaz Camagüey, Cuba. Disponible en: https://www.reduc.edu.cu/ 
Alfonso, D., Villazón, A., Milanes, P., Rodríguez, A., \& Espín, R. (2011). Procedimiento general de rediseño organizacional para mejorar el enfoque a procesos. Ingeniería Industrial, XXXII(3), 238-248. Disponible en: $\quad$ https://www.redalyc.org/articulo. oa? id=360433577010

Alonso, A., Michelena, E., \& Alfonso, D. (2013). Dirección por procesos en la Universidad. Ingeniería Industrial, XXXIV(1), 87-95. Disponible en: http://scielo.sld.cu/scielophp? script=sci_abstract\&pid $=$ S181559362013000100009

Amozarrain, M. (2009). La gestión por procesos. Disponible en: http:// personales.jet.es/amozarrain/Gestion procesos.htm. \%20 \%20 \%20 \%20\%20 POR \%2OQUE \%2OLA \%20GESTION \%20 POR \%2OPROCESOS

Arrascaeta, R. (2005). Enfoque a proceso. Conferencia Digital, Foro Mundial de la Calidad INLAC, Veracruz, México.

Beltrán, J., Carmona, M., Carrasco, R., Rivas, M., \& Tejedor, F. (2009). Guía para una gestión basada en procesos. In I. A. d. Tecnología (Ed.). Disponible en: http:// grupos.emagister.com/ficheros/vcruzada?fdwn=1\&idGrupo=24173\&idFichero $=1081358$

Betancourt, Y. (2018). Pasos metodológicos para la implementación del enfoque basado en proceso en la fábrica de helados Coppelia [Tesis de posgrado]. Universidad de Camagüey "Ignacio Agramonte Loynaz", Camagüey, Cuba. Disponible en: https://www.reduc.edu. $\mathrm{cu} /$

Bravo, J. (2009). Gestión de Procesos. Santiago de Chile: Editorial Evolución S.A. Disponible en: https://silo.tips/download/ gestion-de-procesos-alineados-con-laestrategia

Bravo, J. (2012). Gestión de procesos en Chile. Diagnóstico y propuesta. In E. S.A. (Ed.).
Calderon, L. (2018). Guía metodológica para la implementación del enfoque basado en proceso en ETECSA [Tesis de pregrado]. Universidad de Camagüey "Ignasio Agramonte Loynaz" Camagüey, Cuba. Disponible en: https://www.reduc. edu.cu/

Carrasco, F. (2011). Estudio sobre implementación de gestión basada en proceso en BANCOESTADO [Tesis de posgrado]. Universidad de Chile, Santiago de Chile, Chile. Disponible en: http://repositorio.uchile.cl/ handle/2250/102690

Cejas, C. (2012). Marco teórico referencial para introducir la gestión por proceso. La Habana, Cuba. Disponible en: http://. monografias. com/trabajos94/ basesgenerales-introducir-gestionproceso

Eneque, K. \& Tello, J. (2020). Gestión por proceso paraincrementarlaproductividad en la empresa "COMERCIO INDUSTRIA Y SERVICIOS GMV E.I.R.L. [Tesis de pregrado]. Universidad Señor de Sipán, Pimentel, Perú. Disponible en: https:// hdl.handle.net/20.500.12802/7755

Gimer, I. (2012). Modelo para mejorar la gestión del proceso de posgrado en la CUJAE [Tesis de posgrado]. Instituto Superior Politécnico José Antonio Echeverría (CUJAE), La Habana, Cuba. Disponible en: http://www.e-libro.com/titulos

Hernández, A., Manrique, E., Manrique, N., Medina, A., \& Nogueira, D. (2018). La gestión por procesos, una vía para mejorar la calidad de vida en un hogar de ancianos. Revista Médica Electrónica, 40(2), 258-269. Disponible en: http:// www.revmedicaelectronica.sld.cu/index. php/rme/article/view/2080/html_390

Hernández, A., Nogueira, D., Medina, A., \& Marqués, M. (2013). Inserción de la gestión por procesos en instituciones hospitalarias. Concepción metodológica 
y práctica. Revista de Administração - RAUSP, 48(4), 739 - 756. DOI: https://doi.org/10.5700/rausp1118

ISO/TC 176/SC 2/N 544R2 (2003). Orientación sobre el concepto y uso del "Enfoque basado en procesos" para los sistemas de gestión.

Llanes, M., Isaac, C. L., Moreno, M., \& García, V. (2014). De la gestión por proceso a la gestión integrada por procesos. Ingeniería Industrial, XXXV, 255-264. Disponible en: http://scielo.sld.cu/pdf/rii/ v35n3/rii02314.pdf

Mallar, M. (2010). La gestión por procesos: un enfoque de gestión eficiente. Visión de futuro, 13(1). Disponible en: https://www.redalyc.org/articulo. oa? id=357935475004

Medina, A., Nogueira, D., Hernández-Nariño, A., \& Comas, R. (2019). Procedimiento para la gestión por procesos: métodos y herramientas de apoyo. Ingeniare, Revista chilena de ingeniería, 27(2), 328342. DOI: http://dx.doi.org/10.4067/ S0718-33052019000200328.

Morell, R. (2017). Guía Metodológica para la gestión por proceso con enfoque de gestión de conocimiento en la UEB de cría porcina "Pablo Miguel López" [Tesis de magister]. Universidad de Camagüey "Ignacio Agramonte Loynaz", Camagüey, Cuba.

Narbarte, C., Álvarez, S., Cortés, J., Hervás, R., Girona, L., López, P., \& Sotos, P. (2007). Guía de gestión por procesos. España: Generalitat Valenciana

Pérez, E., Flores, J., Medina, A., Nogueira, D., \& Oviedo, M. (2017). Enfoque de proceso para la reducción de paros de máquina mediante mantenimiento centrado en confiabilidad. Impacto en la economía como rama de las Ciencias Sociales Revista Magazine de las Ciencias, 2(4), 31-42. Ddisponible en: https://revistas. utb.edu.ec/index.php/magazine/article/ view/380

Pérez, J. (2010). Gestión por procesos. Madrid: ESIC Editorial.

Pernett, J. (2004). La Gestión Educativa por Procesos: Guía para su identificación e implementación. Revista MasEducativa, (6), 1-9. Disponible en: https://www.academia.edu/1481357/ La_Gesti\%C3\%B3n_Educativa_por_ Procesos

Ponce, K. (2016). Propuesta de implementación de gestión por proceso para incrementar los niveles de productividad en una empresa textil. [Tesis de pregrado], Universidad peruana de Ciencias Aplicadas, Lima, Perú. Disponible en: https://repositorioacademico.upc.edu. pe/bitstream/handle/10757/620981/ Tesis\%20Textil\%20S.A.C.\%20-\%20 Katherine\% 20Ponce\% 20 Herrera. pdf? sequence $=1$ \&isAllowed $=y$

Ponjuán, G., Villardefrancos, M. \& León, M. (2005). Principios y métodos para el mejoramiento organizacional. La Habana, Cuba.

Quintana, L., \& Hernández, L. (2013). Diseño del proceso de Dirección Estratégica, cambio de un enfoque funcional a enfoque de proceso. Avances, 15(3), 290-298. Disponible en: https://dialnet.unirioja.es/ servlet/articulo?codigo $=5350889$

Rivero, D. (2013). Procedimiento para la implementación del enfoque basado en proceso del sistema de gestión de la calidad. [Tesis de maestría]. Universidad de Camagüey "Ignacio Agramonte Loynaz", Camagüey, Cuba.

Rivero, M. E. (2018). Implementación del enfoque de proceso en la gestión de la revista científica Retos de la Dirección [Tesis de magiter], Universidad de Camagüey "Ignacio Agramonte Loynaz", Camagüey, Cuba. 
Rodríguez, D. (2017). Propuesta de implementación de la gestión por procesen las actividades misionales y de apoyo de la fundación Desayunito Creando Huellas [Tesis de pregrado]. Universidad Católica de Colombia, Bogotá, Colombia. Disponible en: https://repository.ucatolica.edu.co/ handle/10983/23227

Saballo, E. (2005). Procedimiento para realizar estudios de procesos en empresas hoteleras [Tesis de maestría]. Universidad de Camagüey "Ignacio Agramonte Loynaz", Camagüey, Cuba.

Zaratiegui, J. (1999). La gestión por procesos: su papel e importancia en la empresa. Economía Industrial, VI(330), 81-88. Disponible en: https://dialnet.unirioja.es/ servlet/articulo?codigo $=140164$ 